\title{
Electric field-based ionic control of selective separation layers $\dagger$
}

Received 00th January 20xx, Accepted 00th January 20xx
Yan Zhao, ${ }^{\ddagger a}$ Yanling Liu, ${ }^{\ddagger a}, \mathrm{~b}$ Chao Wang, ${ }^{\mathrm{c}}$ Emily Ortega, ${ }^{\mathrm{a}, \mathrm{d}}$ Xiaomao Wang, ${ }^{\mathrm{b}}$ Yuefeng F. Xie, ${ }^{\mathrm{b}}$ Jiangnan Shen, ${ }^{* c}$ Congjie $\mathrm{Gao}^{\mathrm{c}}$ and Bart Van der Bruggen*a, e

DOI: $10.1039 / \times 0 \times x 00000 x$

The application of membrane technology to separate and extract target ions, such as lithium or chlorine has recently seen considerable growth as a research topic, driven by the growing demand for energy and the status of this technology as environmentally-friendly. Based on the alternating current electric field layer-by-layer assembly technology, we report in this work target ionic control membrane surface multilayers with ion channels and ion exchange sites to control selective ion separation in electrodialysis. The reported surface multilayers utilize ion interactions between electric field force and charged groups, and the mechanics of the target ion controlling the construction of ion exchange sites in an oppositely charged polymer layer and target ion channels in a like-charged monomer layer. The resulting membranes act as a strong barrier for multivalent ions, displaying a selective separation efficiency parameter equal to $99.99 \%$ for $\mathrm{PO}_{4}{ }^{3-} / \mathrm{Cl}^{-}$and $99.99 \%$ for $\mathrm{SO}_{4}{ }^{2-} / \mathrm{Cl}^{-}$in the resulting anion exchange membrane, and $83.70 \%$ for $\mathrm{Mg}^{2+} / \mathrm{Li}^{+}$and $64.51 \%$ for $\mathrm{Ca}^{2+} / \mathrm{Li}^{+}$in the resulting cation exchange membrane. Contrary to the initial selective separation efficiency between monovalent ions $(-16.50 \%$ for $\mathrm{Br} / \mathrm{Cl}^{-}$and $-40.00 \%$ for $\left.\mathrm{K}^{+} / \mathrm{Li}^{+}\right)$, the resulting membranes remarkably enhanced the transport of the target ions more easily than other monovalent ions $10.76 \%$ for $\mathrm{Br} / \mathrm{Cl}^{-}$and $-9.10 \%$ for $\mathrm{K}^{+} / \mathrm{Li}^{+}$). This work suggests promising new quantum mechanics of ion and electric field-based ionic control concepts to synthesize membranes with the ability to selectively separate a target ion.

Critical energy shortages and environmental pollution are the most urgent challenges of our modern civilization and have driven scientists to develop various techniques for solving

\footnotetext{
a. Department of Chemical Engineering, KU Leuven, Celestijnenlaan 200F, B-3001 Leuven, Belgium.

b. State Key Joint Laboratory of Environment Simulation and Pollution Control, School of Environment, Tsinghua University, Beijing 100084, China.

c. Center for Membrane Separation and Water Science \& Technology, Ocean College, Zhejiang University of Technology, Hangzhou 310014, P. R. China.

d. Department of Biological Sciences, University of Wisconsin-Milwaukee,

Milwaukee, WI, 53201, United States.

e. Faculty of Engineering and the Built Environment, Tshwane University of Technology, Private Bag X680, Pretoria 0001, South Africa.

†Electronic Supplementary Information (ESI) available. available. See
}

¥These authors contributed equally to this work.

these urgent problems. $\underline{1}, \underline{2}$ Extracting clean resources for energy utilization from the ambient environment is an economically and ecologically friendly way to replace conventional nonrenewable and environmentally costly fossil

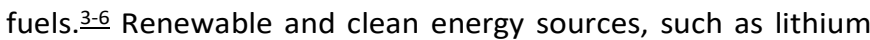
and chlorine ions, which are abundant in salt lakes and sea water, have been regarded as one of the most promising and efficient replacements in recent years. $\underline{7}, \underline{8}$ However, the coexistence of chemically similar ions in salt lakes and sea water severely limits the extraction of high-purity target ions through traditional techniques with low selective efficiency, such as salting out, precipitation, and nanofiltration. $\frac{9-11}{\text { It }}$ has been revealed that biological membrane materials exhibit an extraordinarily efficient selectivity for ion transport through enriched charged residue ion channels. $\underline{12}, \underline{13}$ These discoveries have motivated scientists to explore further to imitate and synthesize artificial materials with ionic transport channels for separation and extraction of target ions. $\underline{14}, \underline{15}$

Naturally, ion exchange membranes (IEMs) with positively or negatively charged functional groups, which function as anion exchange membranes (AEMs) or cation exchange membranes (CEMs), are expected to mimic the ion separation properties found in biological membranes when used in electro-driven processes. $16-18$ Currently, IEMs with selective separation properties for monovalent ions have been explored, which noticeably expands the application field of IEMs and demonstrates their potential in the extraction of ionic resources. $\frac{19}{\text { In }}$ classical theories, the electrostatic repulsion effect between solution ions and the charged membrane surface and the size sieving effect between the hydrated ionic diameter and the membrane structure are the most important rules for preparation of the AEMs/CEMs with selective monovalent ion separation properties. $20-22$ However, based on the electrostatic repulsion effect, the coexistence of a large number of monovalent anions, such as $\mathrm{Cl}^{-}$and $\mathrm{Br}^{-}$, or monovalent cations, such as $\mathrm{Li}^{+}$and $\mathrm{K}^{+}$, with chemically similar properties are difficult to be selectively separated from each other. $\underline{23}$ Furthermore, the size sieving effect may cause an inevitable low desalination efficiency, which leads to a high energy consumption. $\underline{24}$ 
(a)

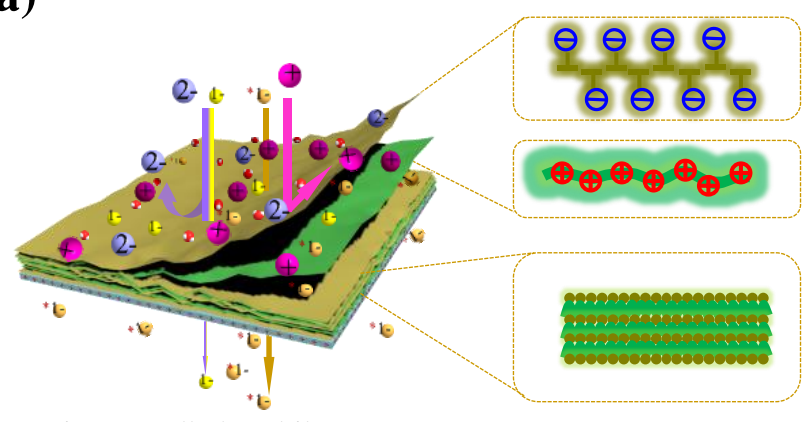

Ionic controlled multilayer AEM

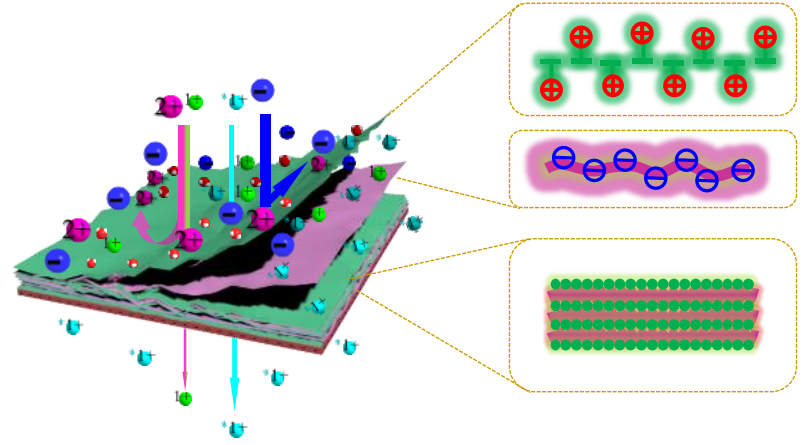

Ionic controlled multilayer CEM (b)
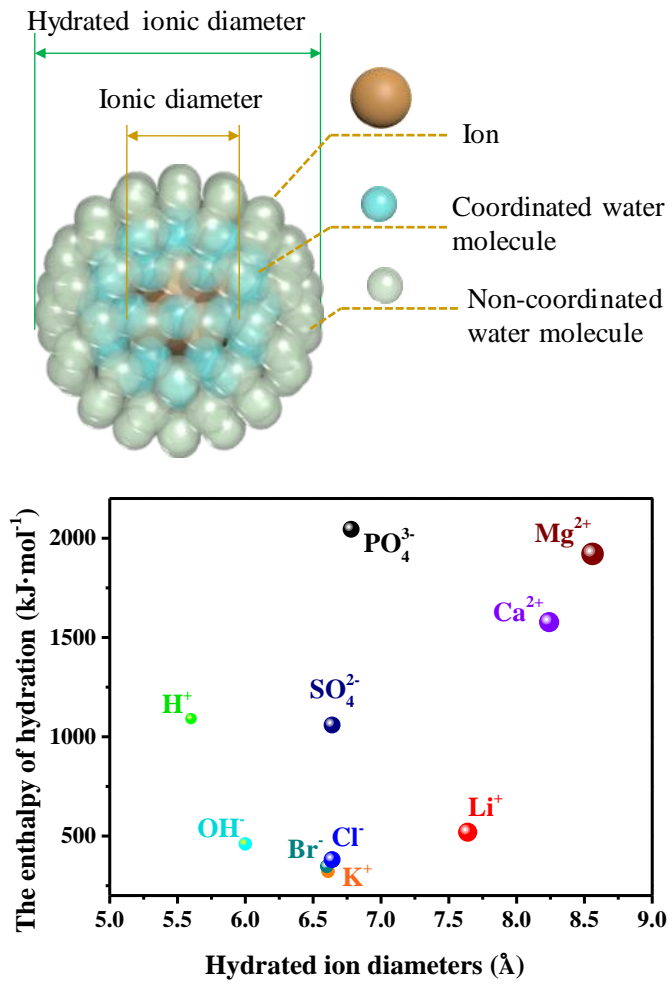

(c)

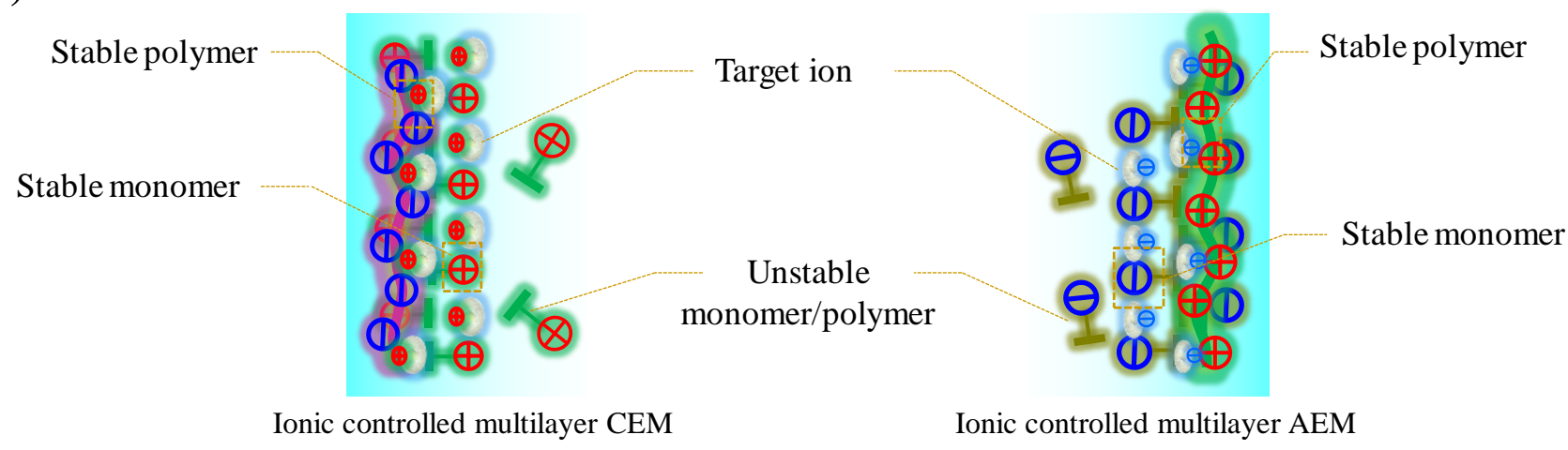

Fig. 1 Design of the ionic-controlled multilayer membranes. (a) Schematic of the design of ionic-controlled multilayer membranes by surface layer-by-layer assembly of the oppositely charged polymer layer and the similarly charged monomer layer. The ioniccontrolled multilayer AEM was synthesized by L-dopamine self-polymerization and amide reaction with 4-aminobenzenesulfonic acid (L-PDA\#ABS) layers, and assembly with HACC layers; it exhibits a dominant repulsion against cations and multivalent anions and enhances the transport of the target anion. The ionic-controlled multilayer CEM was synthesized by dopamine selfpolymerization and reaction with 2, 3-epoxypropyl trimethyl ammonium chloride (PDA\#ETAC) layers, and assembly with PSSMA layers; it exhibits a dominant repulsion against anions and multivalent cations and enhances the transport of the target cation. (b) Schematic of the hydrated ion consists of ion, coordinated and non-coordinated water molecules; relationship between the hydrated ionic diameter and the enthalpy of hydration $\left(-\Delta \mathrm{H}^{\circ}\right)$. (c) Schematic of under the alternating current $(50 \mathrm{~Hz})$, the unstable polymers falling from the membrane surface and the target ion controlling the construction of the special multilayer surface.

In terms of traditional layer-by-layer (LbL) assembly technology, researchers usually focus on adsorption of positively/negatively charged polyelectrolytes on a membrane surface in a perfect manner to enhance the selectivity. $\underline{25}, \underline{26}$ Few reports focus on the preparation of a membrane based on the mechanics of ions and the connection between hydrated ions and the enthalpy of hydration in the electric field when using the LbL assembly technology. Due to the different features of mechanics of ions in an electric field, the use of the 
target ion to control materials with precision ion response has garnered increasing concern in recent years. $27-29$

Based on the mechanics of ions in an electric field, herein, we use an alternating current electric field and a target ion to control the architecture of the multilayer, which has the target ion exchange sites in an oppositely charged polymer layer and the target ion channels in a similarly charged monomer layer. The target ion-controlled multilayer AEMs were synthesized via alternating current LbL assembly technology, $\underline{30}$ using Ldopamine self-polymerization (LPDA) followed by an amide reaction with 4-aminobenzenesulfonic acid (ABS) to form negatively charged monomer layers (LPDA\#ABS) and assembly with 2-hydroxypropyltrimethyl ammonium chloride chitosan (HACC) layers to build a multilayer on the commercial AEM surface (Fig. 1a). Similarly, multilayer CEMs were synthesized by using dopamine self-polymerization (PDA), which then reacted with 2, 3-epoxypropyl trimethyl ammonium chloride (PDA\#ETAC) to form positively charged monomer layers (PDA\#ETAC); assembly with poly(4-styrenesulfonic acid-comaleic acid) sodium salt (PSSMA) layers to build a multilayer on the CEM surface was then completed (Fig. 1a). Because of the specific hydrated ion characteristics in aqueous solution, such as the hydrated ionic diameter and the enthalpy of hydration $\left(-\Delta \mathrm{H}^{\circ}\right)$ (Fig. $1 \mathrm{~b}$ and Fig. S2), each ion shows its specific mechanical properties (Shown in Fig. S1 in Supporting Information). $\underline{27}, \underline{28}, \underline{31} \mathrm{In}$ an electric field, the target hydrated ion may be used to control the special structure of multilayer (Fig. 1c). Under an alternating current field $(50 \mathrm{~Hz})$, the unstable charged monomers and polymers could not be coated on the membrane surface, which ensured the stability of the multilayer (details shown in Fig. S3 in Supporting Information). The resulting membranes with different multilayers were named as AEM-m ( $m$ is the number of bilayers on AEM surface, which $\mathrm{m}=0.0,0.5,1.5,3.5$ and 5.5) and CEM- $\mathrm{n}(\mathrm{m}$ is the number of bilayers on AEM surface, which $n=0.0,0.5,1.5,3.5$ and 5.5).

The monomers' chemical structure with a negatively charged sulfonic group and a positively charged quaternary ammonium group, which constituted the surface functional layer for AEMs and CEMs, respectively, is shown in Fig. 2a, and the other polymer chemical structures is shown in Fig. S4 and S5. To explore the surface charge of the resulting membranes, the $\zeta$ potential was measured from $\mathrm{pH} 3$ to 10 (Fig. $2 \mathrm{~b}$ and $2 \mathrm{c}$ ). The variation of the $\zeta$ potential with the increase of the number of multilayers can be explained by the effect of the positively/negatively charged multilayer. In AEMs (Fig. 2b), increasing the $\mathrm{pH}$ decreased the $\zeta$ potential of the membranes. Compared to AEM-0.0, AEM-0.5 showed a higher variation of the $\zeta$ potential. Furthermore, increasing the number of multilayers (from AEM-0.5 to AEM-5.5) caused the variation range of the $\zeta$ potential to develop in a flat trend. Similarly, CEM-0.5 showed a higher variation of the $\zeta$ potential range than CEM-0.0, and the variation range of the $\zeta$ potential developed in a flat trend from CEM-0.5 to CEM-5.5. This phenomenon occurred due to an increased number of positively/negatively charged multilayers, which endowed the membrane surface with a homogeneous charge property. (a)

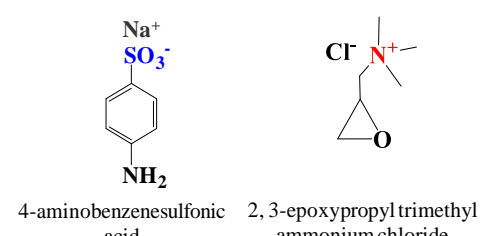

acid ammonium chloride

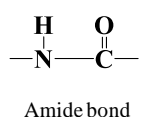

(d)

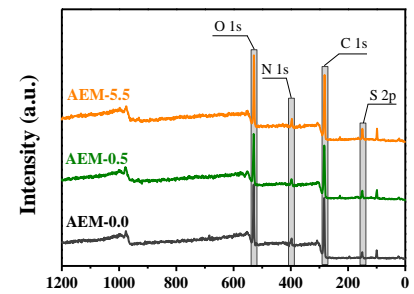

(f) Binding Energy (eV)
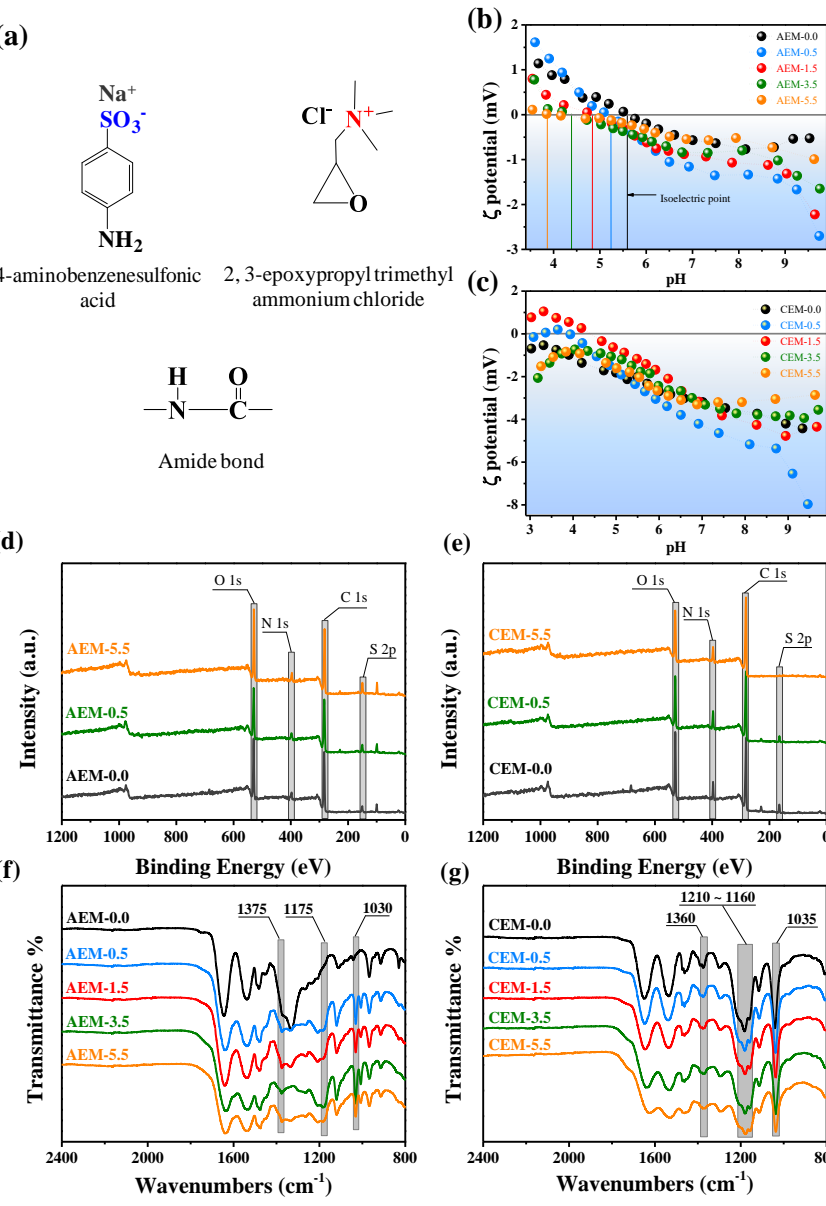

(e)
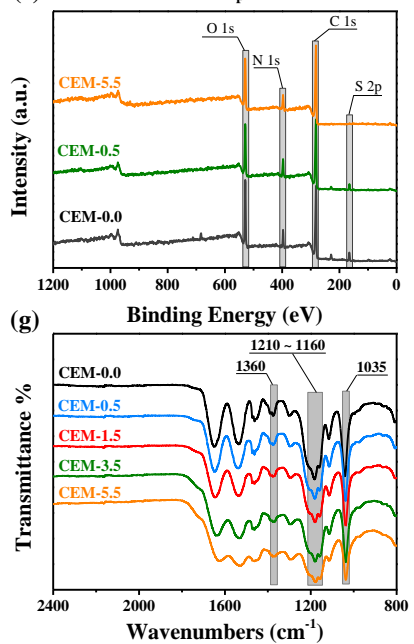

Fig. 2 Monomers for layer-by-layer assembly and characterizations of membrane surface chemistry. (a) The chemical structure of ABS and ETAC. (b) and (c) The zeta potentials of AEMs and CEMs, respectively. (d) and (e) The XPS spectra of membrane surface elements ( $O 1 s, N 1 s, C 1 s$ and $S$ $2 p$ ) in AEMs and CEMs, respectively. (f) and (g) The ATR-FTIR spectra of membrane surface groups change in AEMs and CEMs, respectively.

X-ray photoelectron spectroscopy (XPS; Fig. $2 \mathrm{~d}$ and 2e) and attenuated total reflectance Fourier transform infrared spectroscopy (ATR-FTIR; Fig. $2 \mathrm{f}$ and $2 \mathrm{~g}$ ) of the membranes show newly introduced elements and functional groups on the membrane surface. The peaks located at 528.7, 397.4, 282.3, and $164.8 \mathrm{eV}$ in XPS spectra represent the elements $\mathrm{O} 1 \mathrm{~s}, \mathrm{~N} 1 \mathrm{~s}$, $\mathrm{C} 1 \mathrm{~s}$ and $\mathrm{S} 2 \mathrm{p}$, respectively. $\frac{32-35}{}$ The elemental analysis reveals that by adding multilayers, the $S 2 p$ element in the AEMs also increased (Fig. 2d). Fig. 2e shows that the membrane surface $\mathrm{N}$ 1s element of CEMs was decreased with the increase of the number of multilayers. This is probably because the outside layer consisted of negatively charged sulfonic groups in AEM5.5 and positively charged quaternary ammonium groups in CEM-5.5. That the peaks at 1375, 1175 and $1030 \mathrm{~cm}^{-1}$ of ATRFTIR spectra, which belong to the asymmetric and symmetric stretching vibration of $\mathrm{S}=\mathrm{O}$ and the stretching vibration of $\mathrm{S}-\mathrm{O}$, respectively, are only presented in the as-prepared AEMs confirms that the $S 2 p$ only exists in the sulfonic groups. $\frac{36}{}$ 

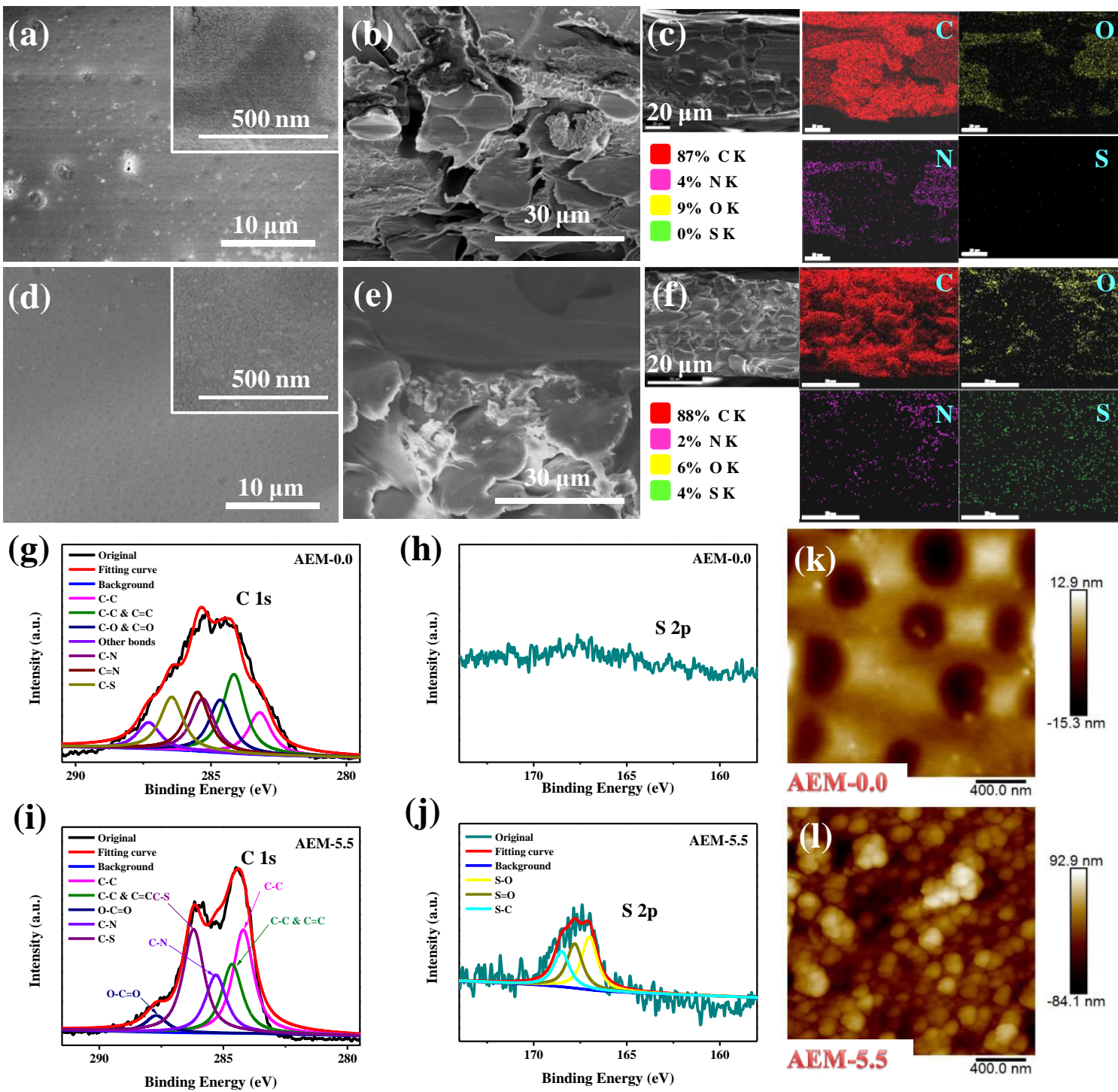

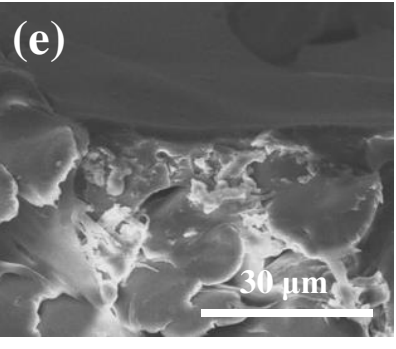

(h)

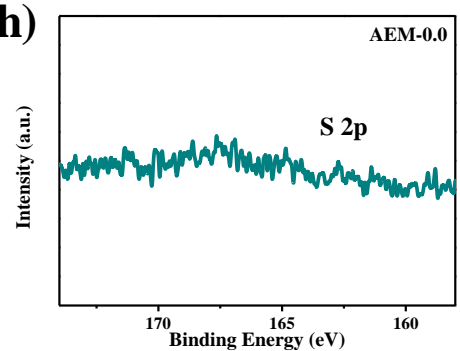

(j)

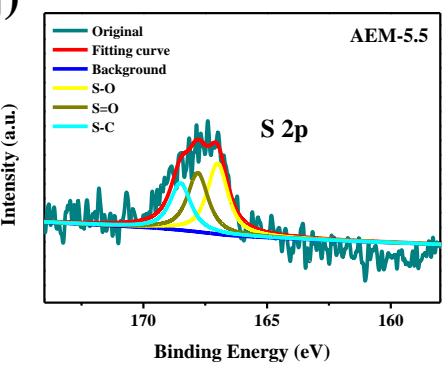

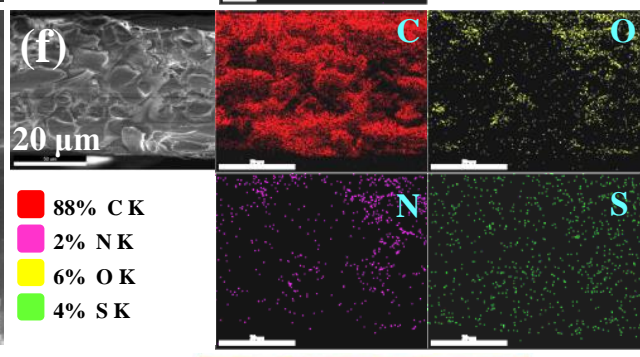
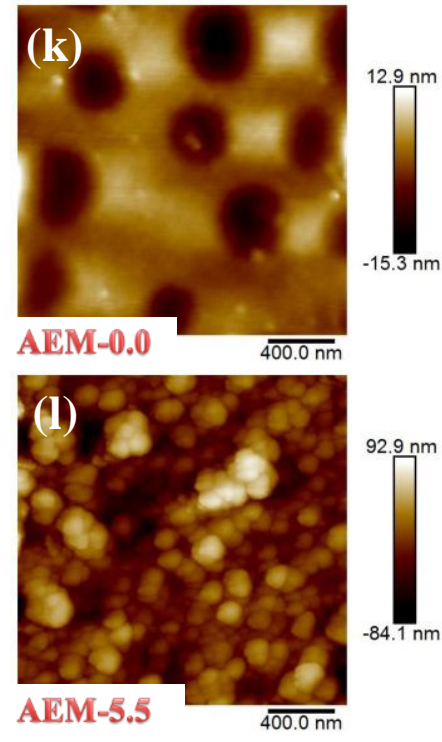

Fig. 3 Scanning electronic microscopic (SEM), XPS and AFM characterization of modified AEMs. (a) Surface, (b) cross-section SEM image and (c) its element mapping of AEM-0.0. (d) Surface, (e) cross-section SEM image and (f) its element mapping of AEM-5.5. The XPS spectra of high resolution ( $g$ ) $C 1 s$ and (h) S 2p of AEM-0.0. The XPS spectra of high resolution (i) C 1s and (j) S 2p of AEM5.5. The AFM surface image of (k) AEM-0.0 and (I) AEM-5.5.

In addition, the peaks at 1360 and $1035 \mathrm{~cm}^{-1}$ are the characteristic peaks of $\mathrm{C}-\mathrm{N}$, and the peak at $1210-1160 \mathrm{~cm}^{-1}$ is the stretching vibration of $\mathrm{C}-\mathrm{O}-\mathrm{C}$, which reflects that the ETCA has grafted onto membrane surface through the reaction of the quaternary ammonium groups. $\frac{37}{3}$

In order to further characterize the resulting AEMs, the surface and cross-section morphology as well as the crosssection element distribution mapping of AEMs were observed by scanning electronic microscopy (SEM; Fig. 3a to $3 f$ and Fig. S8). The uneven surface (Fig. 3a), irregular cross-section with supportive fabric (Fig. 3b) and low S element distribution (Fig. 3c) of AEM-0.0 can be clearly observed. Furthermore, the homogeneous sphere shapes along the surface (Fig. 3d) and the surface multilayer of AEM-5.5 can be distinguished from the SEM cross-section image (Fig. 3f). In addition, elemental S was widely distributed in AEM-5.5 (Fig. 3f). The high resolution XPS spectra of $C 1 s$ and $S 2 p$ further illustrated that the chemical component of membrane surface had been modified. Unlike AEM-0.0 (Fig. $3 \mathrm{~g}$ and $3 \mathrm{~h}$ ), in the high resolution C $1 \mathrm{~s}$ XPS spectra of AEM-5.5, a new peak appeared at $286.4 \mathrm{eV}$, which is indicative of C-S vibration (Fig. 3i). The high resolution S $2 p$ XPS spectra (Fig. 3j) displayed peaks at $167.4 \mathrm{eV}$ (S-O vibration), $168.0 \mathrm{eV}$ (S=O vibration), and $168.8 \mathrm{eV}$ (S-C vibration). This suggests that the membrane surface has been assembled with negatively charged sulfonated groups. Through characterization by atomic force microscopy (AFM), the amount of concave section distribution of AME-0.0 (Fig. 3k) can be compared to that of AEM-5.5 (Fig. 3I), which showed homogeneous sphere shapes indicative of atomic force property and an enlarged membrane specific surface area. 
Based on the Wenzel model theory, since the multilayers were comprised of hydrophilic materials and the surface roughness increased, the final hydrophilicity will be enhanced. $\underline{38}$

The irregular and uneven surface with supportive fabric can be also observed from the surface (Fig. 4a) and crosssection (Fig. 4b) SEM images of CEM-0.0. The element mappings from the SEM cross-section image reveal that CEM0.0 contains little elemental $\mathrm{N}$ (Fig. 4c). Fig. 4d shows the homogeneous sphere shapes in the surface of CEM-5.5. The homogeneous multilayer on the membrane surface can be clearly distinguished from the cross-section SEM images (Fig. $4 \mathrm{e})$, and its mappings illustrate that the multilayer contains a higher amount of elemental $\mathrm{N}$ (Fig. 4f) (Other SEM images are shown in Fig. S9). Compared to the high resolution XPS spectra of $C$ 1s in CEM-0.0 (Fig. 4g), that of CEM-5.5 shows a higher proportion of $\mathrm{C}-\mathrm{N}$ bonds (Fig. 4i). The high resolution $\mathrm{N}$ 1s XPS spectra of CEM-0.0 can only be fit into the two peaks at 399.4 $\mathrm{eV}$ (primary ammonium N-C vibration) and $400.0 \mathrm{eV}$ (secondary ammonium $\mathrm{N}-\mathrm{C}$ vibration) (Fig. 4h). However, a new peak appeared at $401.85 \mathrm{eV}$ in the high resolution $\mathrm{N}$ 1s XPS spectra of CEM-5.5, which results from the tertiary ammonium vibration (Fig. $4 \mathrm{j}$ ). This reflects that the membrane surface multilayer was assembled with positively charged quaternary ammonium groups. The AFM images of CEM-0.0 (Fig. 4k) and CEM-5.5 (Fig. 4I) also illustrate that electric fieldbased ionic control results in a multilayer with higher hydrophilicity and lager specific surface area.

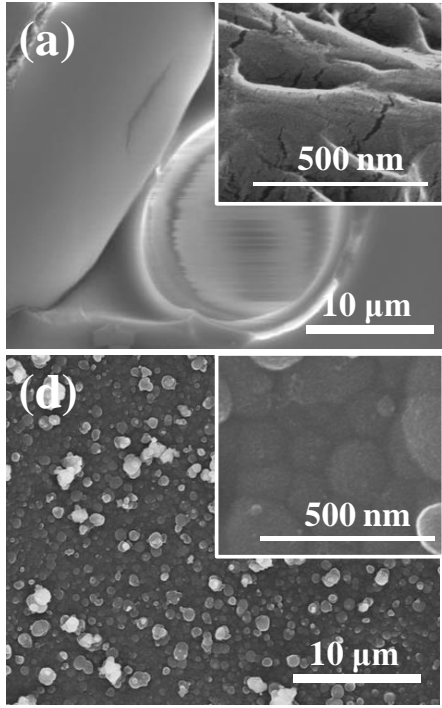

(g)

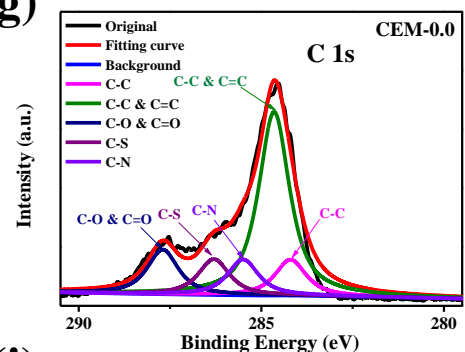

(i)

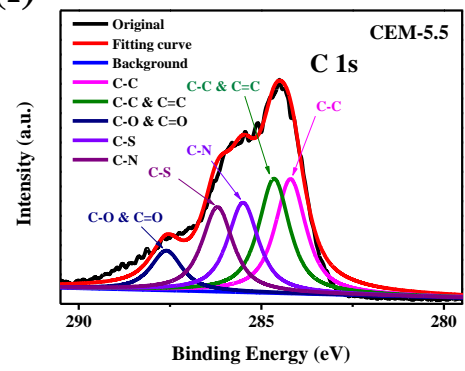

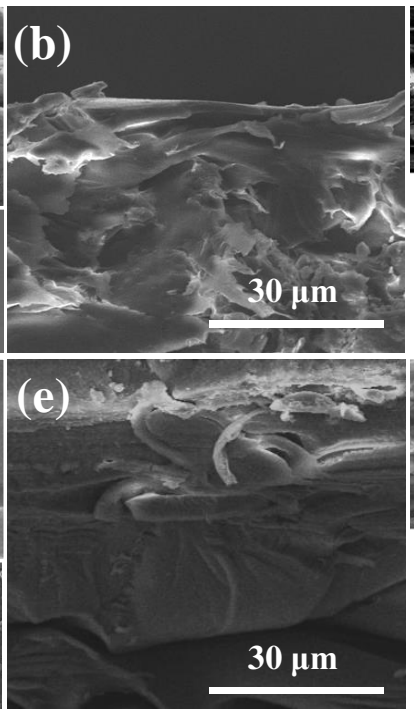
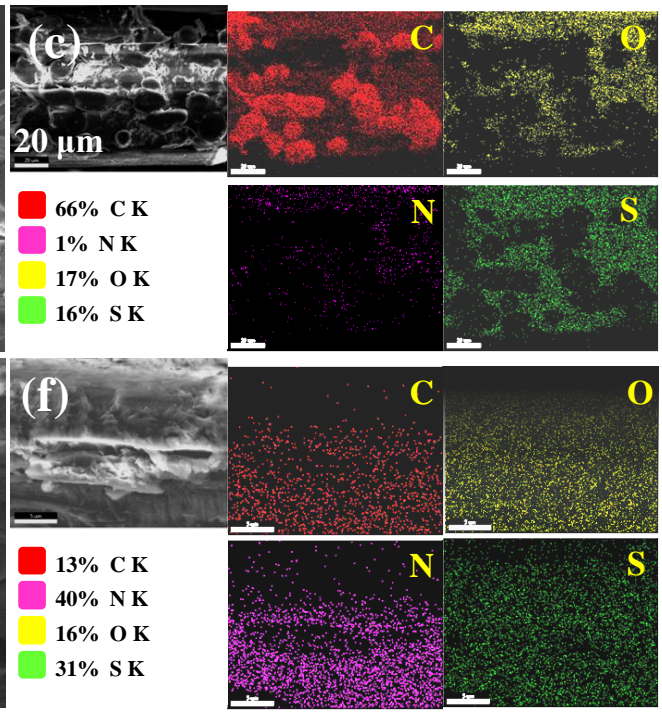

(h)

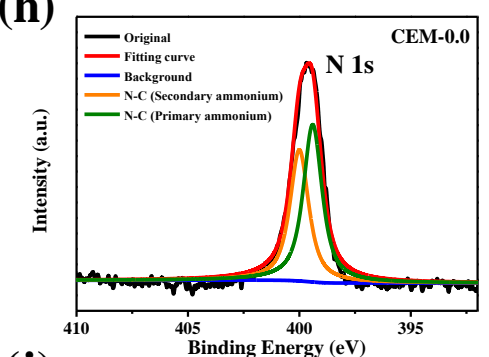

(j)

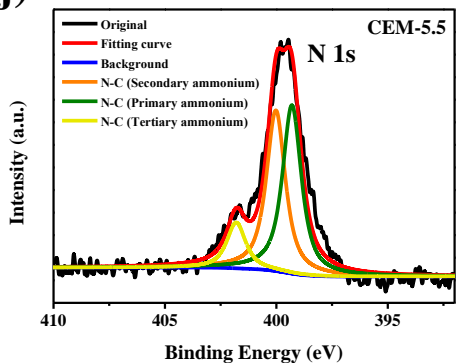

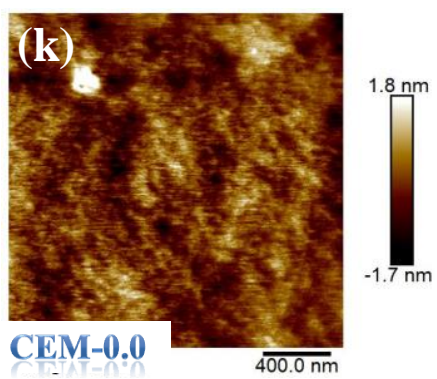

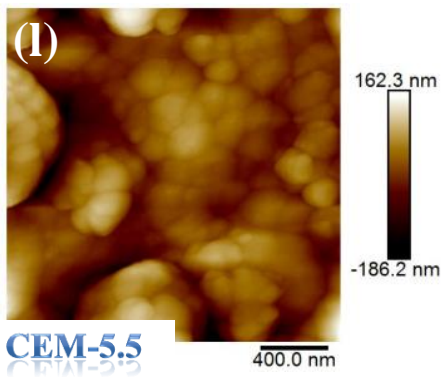

Fig. 4 Scanning electronic microscopic (SEM), XPS and AFM characterization of modified CEMs. (a) Surface, (b) cross-section SEM image and (c) its element mapping of CEM-0.0. (d) Surface, (e) cross-section SEM image and (f) its element mapping of CEM-5.5. The XPS spectra of high resolution ( $g$ ) C $1 s$ and $(h) S 2 p$ of CEM-0.0. The XPS spectra of high resolution (i) C $1 s$ and (j) S $2 p$ of AEM5.5. The AFM surface image of ( $k$ ) CEM-0.0 and (I) CEM-5.5. 
(a)

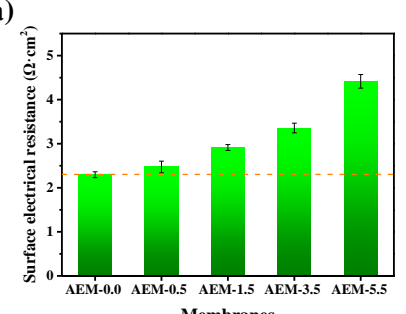

(c)

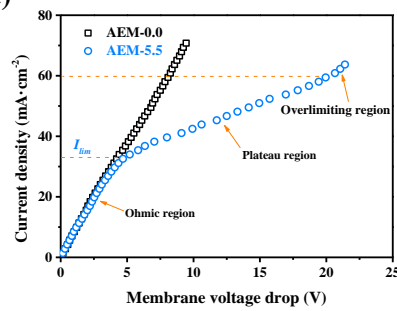

(b)

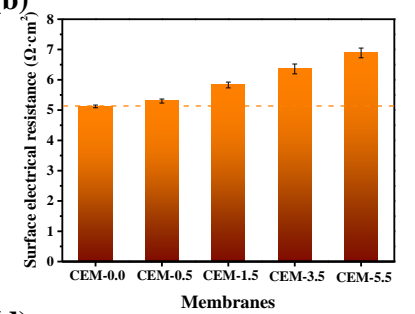

(d)

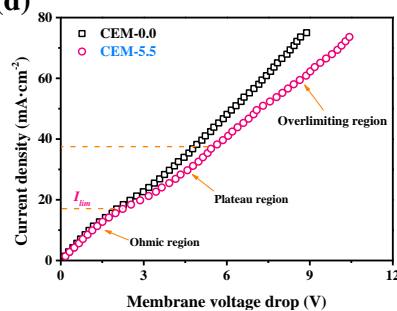

Fig. 5 Electrochemistry of the prepared membranes. The surface electrical resistance of (a) AEMs and (b) CEMs. The current-voltage curve of (c) AEM-0.0 and AEM-5.5 and (d) CEM-0.0 and CEM-5.5.

The membrane surface electrical resistances were measured by a membrane-based device (Fig. S6, see details in Supporting Information). All the resulting membranes show a higher surface electrical resistance than the pristine one. However, compared to AEM-0.0, of which the surface electrical resistance is $2.30 \Omega \cdot \mathrm{cm}^{2}$, the surface electrical resistance values of AEM-0.5, AEM-1.5, AEM-3.5 and AEM-5.5 increase by $0.17,0.61,1.05$ and $1.99 \Omega \cdot \mathrm{cm}^{2}$, respectively (Fig. 5a). Similarly, compared to CEM-0.0 (Fig. 5b), of which the surface electrical resistance is $5.13 \Omega \cdot \mathrm{cm}^{2}$, the surface electrical resistance values of CEM-0.5, CEM-1.5, CEM-3.5 and CEM-5.5 increase by $0.16,0.68,1.22$ and $1.75 \Omega \cdot \mathrm{cm}^{2}$, respectively. According to the multilayer preparation processes, under the alternating current electric field, the unstable and redundant monomers/polymers cannot be synthesized into the multilayer, thereby keeping the multilayer homogeneous and stable, and the increased surface electrical resistances were not very high. The small increase of surface electrical resistance indicates the low energy requirements in industrial application.

The current-voltage curve, which reflects the electrochemical performance between the membrane potential and the current in desalination process, was tested for a membrane surface area of $20.0 \mathrm{~cm}^{2}$ in $0.1 \mathrm{M} \mathrm{NaCl}$ (Fig. S7, see details in Supporting Information). All the membranes showed three kinds of typical electrochemical behaviors of IEMs, which are the Ohmic region, plateau region and overlimiting region (Fig. 5c, 5d, S12 and S13). When the multilayer number increased to 5.5 bilayers, the limiting current density of AEM-5.5 was about $34.0 \mathrm{~mA} \cdot \mathrm{cm}^{-2}$ (Fig. 5c) and that of CEM-5.5 was $18.5 \mathrm{~mA} \cdot \mathrm{cm}^{-2}$ (Fig. $5 \mathrm{~d}$ ). The resulting membranes were compared to commercial monovalent selective membranes (ASV and CSO) for which the limiting current densities were $28.0 \mathrm{~mA} \cdot \mathrm{cm}^{-2}$ and $15.0 \mathrm{~mA} \cdot \mathrm{cm}^{-2}$, respectively (Fig. S14). The above results indicate the

(a)

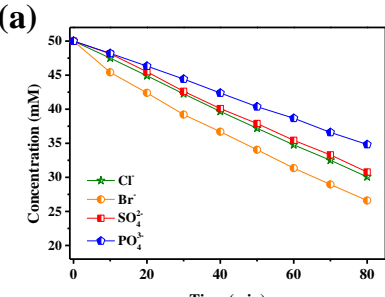

(c)

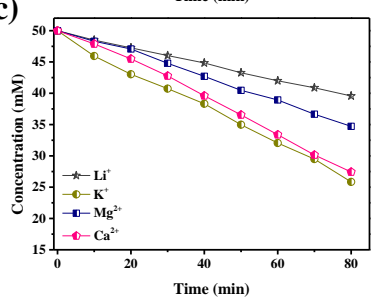

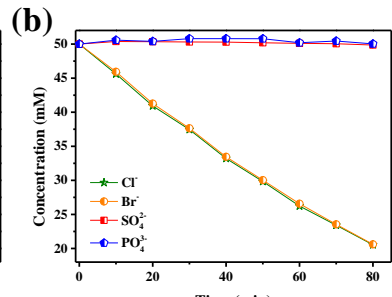

(d)

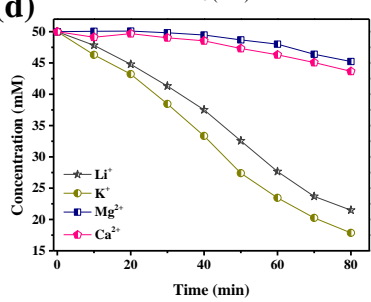

Fig. 6 Comparison of ion selective separation performances. The selective separation performance for $\mathrm{Cl}^{-}, \mathrm{Br}^{-}, \mathrm{SO}_{4}{ }^{2-}$ and $\mathrm{PO}_{4}{ }^{3-}$ of (a) AEM-0.0 and (b) AEM-5.5. The selective separation performance for $\mathrm{Li}^{+}, \mathrm{K}^{+}, \mathrm{Mg}^{2+}$ and $\mathrm{Ca}^{2+}$ of (c) CEM-0.0 and (d) CEM-5.5.

promising electrochemical performance of the resulting membranes.

To explore the separation performance of the resulting membranes for the selective target ion, as shown in Fig. S15a and Fig. S16a (all the initial ions were $50 \mathrm{mM}$, and the initial voltage was $15.0 \mathrm{~V}$ for the $20 \mathrm{~cm}^{2}$ of membrane), we investigated the selective separation of $\mathrm{Cl}^{-}$of the AEMs in ED by using a model saline solution containing $\mathrm{NaCl}, \mathrm{NaBr}, \mathrm{Na}_{2} \mathrm{SO}_{4}$ and $\mathrm{Na}_{3} \mathrm{PO}_{4}$ (Fig. S15, Fig. 6a and 6b) and also investigated the selective separation of $\mathrm{Li}^{+}$through the CEMs in ED by using a model saline solution containing $\mathrm{KCl}, \mathrm{LiCl}, \mathrm{MgCl}_{2}$ and $\mathrm{CaCl}_{2}$ (Fig. S16, Fig. $6 \mathrm{c}$ and $6 \mathrm{~d}$ ). As discussed before (Fig. 1b), the hydrated ionic diameters of $\mathrm{Br}^{-}, \mathrm{Cl}^{-}, \mathrm{SO}_{4}{ }^{2-}$ and $\mathrm{PO}_{4}{ }^{3-}$ are similar $(0.660$, $0.664,0.664$ and $0.678 \mathrm{~nm}$, respectively), so that the concentrations of these ions maintain a similar decreasing trend in the diluate cell for AEM-0.0. The enthalpy of hydration $\left(-\Delta \mathrm{H}^{\circ}\right)$ for $\mathrm{Br}^{-}, \mathrm{Cl}^{-}, \mathrm{SO}_{4}{ }^{2-}$ and $\mathrm{PO}_{4}{ }^{3-}$ is $347,381,1059$ and 2025 $\mathrm{kJ} \cdot \mathrm{mol}^{-1}$, respectively, which means that $\mathrm{SO}_{4}{ }^{2-}$ and $\mathrm{PO}_{4}{ }^{3-}$ need more energy to transfer through the membrane. Thus, the transport behavior of hydrated ions through AEM-0.0 is $\mathrm{Br}^{-} \geq$ $\mathrm{Cl}^{-} \geq \mathrm{SO}_{4}^{2-} \geq \mathrm{PO}_{4}{ }^{3-}$ (Fig. 6a). Compared to AEM-0.0, AEM-5.5 shows that $\mathrm{SO}_{4}{ }^{2-}$ and $\mathrm{PO}_{4}{ }^{3-}$ can hardly cross the membrane and the transport behavior for $\mathrm{Cl}^{-}$is enhanced (Fig. 6b). These abnormal phenomena probably occur because the target ionic control multilayer showed a high electrostatic repulsion for multivalent ions and constructed a $\mathrm{Cl}^{-}$channel for enhancing $\mathrm{Cl}^{-}$transport. A similar conclusion was drawn for the CEMs. The order of hydrated ionic diameters for cations is $\mathrm{Mg}^{2+}>\mathrm{Ca}^{2+}>$ $\mathrm{Li}^{+}>\mathrm{K}^{+}$with values of $0.856,0.824,0.764$ and $0.662 \mathrm{~nm}$, respectively, and the enthalpy of hydration $\left(-\Delta H^{\circ}\right)$ is 1921 , 1577, 519 and $322 \mathrm{~kJ} \cdot \mathrm{mol}^{-1}$, respectively. The transport behavior for CEM-0.0 is $\mathrm{K}^{+}>\mathrm{Ca}^{2+}>\mathrm{Mg}^{2+}>\mathrm{Li}^{+}$(Fig. 6c). Due to the smallest values of hydrated ionic diameter and enthalpy of hydration $\left(-\Delta \mathrm{H}^{\circ}\right), \mathrm{K}^{+}$transports the membrane faster than others. Moreover, multivalent cations have a stronger electric 
(a)

(c)
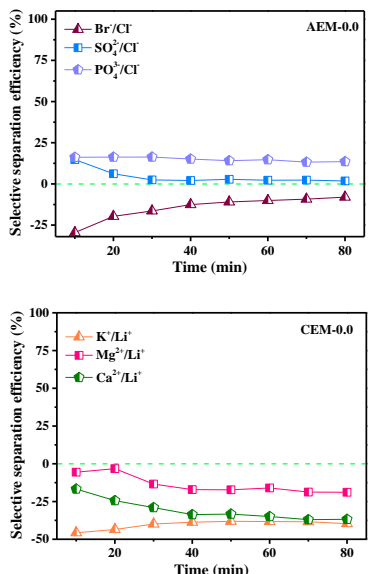

(e)

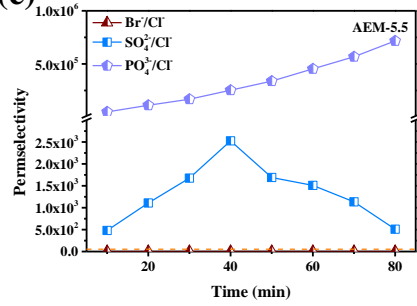

(b)

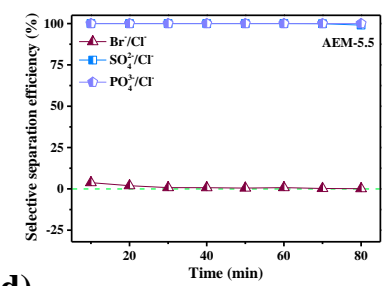

(d)

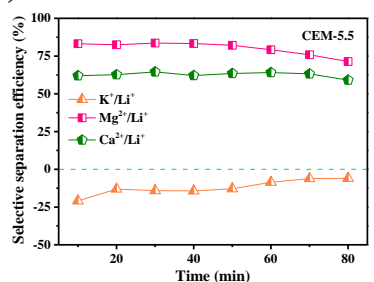

(f)

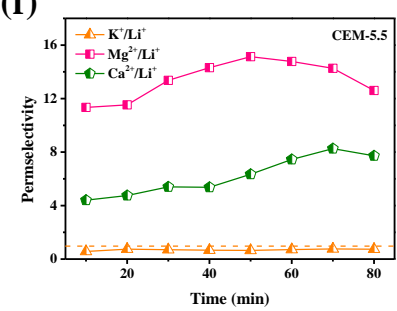

Fig. 7 Selective separation efficiency and permselectivity behavior. The selective separation efficiency of (a) AEM-0.0, (b) AEM-5.5, (c) CEM-0.0 and (d) CEM-5.5. The permselectivity of (e) AEM-5.5 and (f) CEM-5.5.

field force than monovalent cations, the transport behavior of $\mathrm{Mg}^{2+}$ and $\mathrm{Ca}^{2+}$ is greater than that of $\mathrm{Li}^{+}$. When the CEM surface was coated with the resulting multilayers, CEM-5.5 became a strong barrier for $\mathrm{Mg}^{2+}$ and $\mathrm{Ca}^{2+}$ ions through electrostatic repulsion, and the transport behavior of $\mathrm{Li}^{+}$was greatly enhanced due to the constructed $\mathrm{Li}^{+}$channels and ion exchange sites (Fig. 6d).

The selective separation efficiency parameter, which is the retention or separation parameter between two different ions, is used to show the transport rate of target ion. AEM-0.0 was found to have a selective separation efficiency parameter of $\mathrm{Cl}^{-}$ (in $30 \mathrm{~min}$ ) equal to $2.41 \%\left(\mathrm{SO}_{4}{ }^{2-} / \mathrm{Cl}\right), 16.22 \%\left(\mathrm{PO}_{4}{ }^{3-} / \mathrm{Cl}^{-}\right)$and $16.50 \%\left(\mathrm{Br}^{-} / \mathrm{Cl}^{-}\right)$(Fig. 7a). In comparison, the same parameter for AEM-5.5 is $99.99 \%$ for $\mathrm{SO}_{4}{ }^{2-} / \mathrm{Cl}^{-}, 99.99 \%$ for $\mathrm{PO}_{4}{ }^{3-} / \mathrm{Cl}^{-}$and $0.76 \%$ for $\mathrm{Br}^{-} / \mathrm{Cl}^{-}$in $30 \mathrm{~min}$ (Fig. 7b). The construction of a strong barrier for multivalent ions caused $\mathrm{PO}_{4}{ }^{3-}$ and $\mathrm{SO}_{4}{ }^{2-}$ to be rejected by the multilayer, and the improved selective separation efficiency parameter of $\mathrm{Br}^{-} / \mathrm{Cl}^{-}$suggests that the transport behavior for $\mathrm{Cl}^{-}$in ED was enhanced. For CEMs, the selective separation efficiency parameter of $\mathrm{Li}^{+}$(in $30 \mathrm{~min}$ ) by CEM-5.5 is $83.70 \%\left(\mathrm{Mg}^{2+} / \mathrm{Li}^{+}\right), 64.51 \%\left(\mathrm{Ca}^{2+} / \mathrm{Li}^{+}\right)$and $-9.10 \%$ $\left(\mathrm{K}^{+} / \mathrm{Li}^{+}\right)$(Fig. 7c), while for CEM-0.0 values of $-14.45 \%,-29.10 \%$ and $-40.00 \%$ were calculated (Fig. 7d). This strong barrier for $\mathrm{Mg}^{2+}$ and $\mathrm{Ca}^{2+}$ and the enhanced $\mathrm{Li}^{+}$transport property could be used to extract lithium as a resource. Furthermore, AEM5.5 and CEM-5.5 exhibited a much higher permselectivity against multivalent ions than AEM-0.0 and CEM-0.0, respectively. The permselectivity of $\mathrm{PO}_{4}{ }^{3-} / \mathrm{Cl}^{-}$and $\mathrm{SO}_{4}{ }^{2-} / \mathrm{Cl}^{-}$was

as high as 717,000 and 2530 (Fig. 7e), and the permselectivity of $\mathrm{Mg}^{2+} / \mathrm{Li}^{+}$and $\mathrm{Ca}^{2+} / \mathrm{Li}^{+}$is 15 and 8, respectively (Fig. 7f). Evidently, the resulting membranes act as barriers for multivalent ions and render an enhanced transport for target ions.

According to the classical mechanics of ion motion, the focus is usually on the electric field effect in which ions move across IEMs in a perfect manner. The electrostatic repulsion effect and the size sieving effect are the most important mechanisms for membranes with selective monovalent ion separation performance. In this work, the strong barrier for multivalent ions of resulting membranes confirmed these normal phenomena. However, an atypical phenomenon of enhanced transport of a target ion can be obtained by the preparation of target ion exchange sites and target ion channels. In this work, an alternating current electric field (50 $\mathrm{Hz}$ ) was applied in the $\mathrm{LbL}$ assembly process. The quantum state of a microparticle/ion can be described by a wave function $\varphi(\overrightarrow{\mathbf{r}}, \mathrm{t})$, which is the Schrödinger equation:

$$
\mathrm{i} \hbar \frac{\partial}{\partial t} \varphi(\overrightarrow{\mathrm{r}}, \mathrm{t})=\left(-\frac{\hbar^{2}}{2 \mathrm{~m}} \nabla^{2}+\mathrm{V}\right) \varphi(\overrightarrow{\mathrm{r}}, \mathrm{t})
$$

where $\mathrm{m}$ is the weight of a microparticle/ion; $\hbar$ is the reduced Planck constant, which is $1.05 * 10^{-34} \mathrm{~J} \cdot \mathrm{s} ; \nabla$ is the gradient operator, which is

$$
\widehat{p}=-i \hbar \vec{\nabla}
$$

$\widehat{p}$ is the momentum operation

$$
\overline{\overrightarrow{\mathrm{P}}}=\int \varphi^{*}(\overrightarrow{\mathrm{r}}) \widehat{\mathrm{p}} \varphi(\overrightarrow{\mathrm{r}}) \mathrm{d}^{3} \mathrm{x}
$$

The general state of a free microparticle/ion is the formation of a wave packet, which is the superposition of many plane monochromatic waves.

$$
\begin{gathered}
\mathrm{i} \hbar \frac{\partial}{\partial t} \varphi(\overrightarrow{\mathrm{r}}, \mathrm{t})=-\frac{\hbar^{2}}{2 \mathrm{~m}} \nabla^{2} \varphi(\overrightarrow{\mathrm{r}}, \mathrm{t}) \\
\varphi(\overrightarrow{\mathrm{r}}, \mathrm{t})=\frac{1}{(2 \pi \hbar)^{3 / 2}} \int_{-\infty}^{+\infty} \varphi(\overrightarrow{\mathrm{p}}) \exp [\mathrm{i}(\overrightarrow{\mathrm{p}} \cdot \overrightarrow{\mathrm{r}}-\mathrm{Et}) / \hbar] \mathrm{d}^{3} \mathrm{p}
\end{gathered}
$$

due to the relationship between the energy $E$ and the momentum $\overrightarrow{\mathrm{p}}$

$$
\mathrm{E}=\frac{\overrightarrow{\mathrm{p}}^{2}}{2 \mathrm{~m}}
$$

when the potential field without the time $(t)$, then

$$
\varphi(\overrightarrow{\mathrm{r}}, \mathrm{t})=\varphi(\overrightarrow{\mathrm{r}}) \mathrm{f}(\mathrm{t})
$$

thus,

$$
\varphi(\overrightarrow{\mathrm{r}})=\frac{1}{(2 \pi \hbar)^{3 / 2}} \int_{-\infty}^{+\infty} \varphi(\overrightarrow{\mathrm{p}}) \exp \left[\mathrm{i}\left(\overrightarrow{\mathrm{p}} \cdot \frac{\overrightarrow{\mathrm{r}}}{\hbar}\right) \mathrm{d}^{3} \mathrm{p}\right.
$$


Thus, once $\varphi(\vec{r})$ is a determined value, the distribution probabilities of observed values of the mechanical quantity are ensured, which can be used to describe the quantum mechanics of a microparticle/ion in three-dimensional state. Under the electric field, the target ion has its specific coherent superposition of waves and the wave function, so that the target ions are able to control the architecture of the multilayer, which has the target ion exchange sites in oppositely charged polymer layer and the target ion channels in the similarly charged monomer layer.

\section{Conclusions}

In conclusion, target ionic control membrane surface multilayers were created based on ionic control principle and alternating current layer-by-layer assembly technology. The asprepared AEM-5.5 not only has a significantly improved barrier function for multivalent ions, of which the selective separation efficiency parameter is $99.99 \%$ of $\mathrm{PO}_{4}{ }^{3-} / \mathrm{Cl}^{-}$and $99.99 \%$ of $\mathrm{SO}_{4}{ }^{2-}$ $/ \mathrm{Cl}^{-}$, but also has the advantage of $\mathrm{Cl}^{-}$controlled construction of ion exchange sites and $\mathrm{Cl}^{-}$channels improving the selective separation efficiency parameter of $\mathrm{Br} / \mathrm{Cl}^{-}$from $-16.50 \%$ to $0.76 \%$. In addition, CEM-5.5 had a selective separation efficiency parameter as high as $83.70 \%$ for $\mathrm{Mg}^{2+} / \mathrm{Li}^{+}$and $64.51 \%$ for $\mathrm{Ca}^{2+} / \mathrm{Li}^{+}$and enhanced the $\mathrm{Li}^{+}$transport behavior between monovalent cations $\left(\mathrm{K}^{+} / \mathrm{Li}^{+}\right)$from $-40.00 \%$ to $-9.10 \%$. This electric field-based ionic control of selective separation layers shows great promise when applied to the fabrication of various other functional ion exchange membranes for extracting clean ion resources.

\section{Conflicts of interest}

There are no conflicts to declare.

\section{Acknowledgements}

This work was supported by the National Natural Science Foundation of China (No. 21676249 and No. 21878273), and the Project for Assistance of Qinghai from Science Technology Department of Zhejiang Province (No. 2018C26004). Yan Zhao and Yanling Liu would like to acknowledge the support provided by the China Scholarship Council (CSC) of the Ministry of Education, P. R. China (CSC NO. 201708330281 and NO. 201806210110).

\section{Notes and references}

1 M. Elimelech, W. A. Phillip, Science, 2011, 333, 712-717.

2 T. Janoschka, N. Martin, U. Martin, C. Friebe, S. Morgenstern, H. Hiller, M. D. Hager and U. S. Schubert, Nature, 2015, 527, 78-81.

3 H. Liu, C. Chen, G. Chen, Y. Kuang, X. Zhao, J. Song, C. Jia, X. Xu, E. Hitz, H. Xie, S. Wang, F. Jiang, T. Li, Y. Li, A. Gong, R. Yang, S. Das and L. Hu, Adv. Energ. Mater., 2018, 8, 1701616.
4 M. L. Feng, D. Sarma, X. H. Qi, K. Z. Du, X. Y. Huang and M. G. Kanatzidis, J. Am. Chem. Soc., 2016, 138, 12578-12585.

5 L. Cao, W. Guo, W. Ma, L. Wang, F. Xia, S. Wang, Y. Wang, L. Jiang and D. Zhu, Energ. Environ. Sci., 2011, 4, 2259.

6 E. Serrano, G. Rus and J. García-Martínez, Renew. Sust. Energ. Rev., 2009, 13, 2373-2384.

7 H. Che, S. Chen, Y. Xie, H. Wang, K. Amine, X.-Z. Liao and Z.-F. Ma, Energ. Environ. Sci., 2017, 10, 1075-1101.

8 F. Li, X. Jiang, J. Zhao and S. Zhang, Nano Energ., 2015, 16, 488-515.

9 .Zhao, W. Shi, B. Van der Bruggen, C. Gao and J. Shen, Adv. Mater. Interfaces, 2018, 5, 1701449.

10 Y. Guo, Y. Ying, Y. Mao, X. Peng, B. Chen, Angew. Chem. Int. Edit., 2016, 55, 15120-15124.

11 Y. Zhao, C. Zhou, J. Wang, H. Liu, Y. Xu, J. W. Seo, J. Shen, C. Gao and B. Van der Bruggen, J. Mater. Chem. A, 2018, 6, 18859-18864.

12 R. H. Tunuguntla, R. Y. Henley, Y.-C. Yao, T. A. Pham, M. Wanunu, A. Noy, Science, 2017, 357, 792-796.

13 P. Chen, H. Yue, X. Zhai, Z. Huang, G.-H. Ma, W. Wei, L.-T. Yan, Science Adv., 2019, 5, eaaw3192.

14 M. Zhang, K. Guan, Y. Ji, G. Liu, W. Jin and N. Xu, Nat. Commun., 2019, 10, 1253.

15 Y. Zhao, J. Zhu, J. Li, Z. Zhao, S. I. Charchalac Ochoa, J. Shen, C. Gao and B. Van der Bruggen, ACS Appli. Mater. Interfaces, 2018, 10, 18426-18433.

16 T. Xu, J. Membrane Sci., 2005, 263, 1-29.

17 C. Vogel and J. Meier-Haack, Desalination, 2014, 342, 156174.

18 J. Ran, L. Wu, Y. He, Z. Yang, Y. Wang, C. Jiang, L. Ge, E. Bakangura and T. Xu, J.Membrane Sci., 2017, 522, 267-291.

19 Y. Zhao, K. Tang, H. Liu, B. Van der Bruggen, A. Sotto Díaz, J. Shen and C. Gao, J. Membrane Sci., 2016, 520, 262-271.

20 Y. Zhao, K. Tang, H. Ruan, L. Xue, B. Van der Bruggen, C. Gao and J. Shen, J. Membrane S., 2017, 536, 167-175.

21 H. Strathmann, Desalination, 2010, 264, 268-288.

22 Y.-L. Ji, Q.-F. An, Y.-S. Guo, W.-S. Hung, K.-R. Lee and C.-J. Gao, J. Mater. Chem. A, 2016, 4, 4224-4231.

23 Y. Zhao, Y. Li, S. Yuan, J. Zhu, S. Houtmeyers, J. Li, R. Dewil, C. Gao and B. Van der Bruggen, J. Mater. Chem. A, 2019, 7, 6348-6356.

24 C. H. Park, S. Y. Lee, D. S. Hwang, D. W. Shin, D. H. Cho, K. H. Lee, T. W. Kim, T. W. Kim, M. Lee, D. S. Kim, C. M. Doherty, A. W. Thornton, A. J. Hill, M. D. Guiver and Y. M. Lee, Nature, 2016, 532, 480-483.

25 S. Abdu, M. C. Marti-Calatayud, J. E. Wong, M. GarciaGabaldon and M. Wessling, ACS Appl. Mater. Interfaces, 2014, 6, 1843-1854.

26 N. White, M. Misovich, A. Yaroshchuk and M. L. Bruening, ACS Appl. Mater. Interfaces, 2015, 7, 6620-6628.

27 L. Chen, G. Shi, J. Shen, B. Peng, B. Zhang, Y. Wang, F. Bian, J. Wang, D. Li, Z. Qian, G. Xu, G. Liu, J. Zeng, L. Zhang, Y. Yang, G. Zhou, M. Wu, W. Jin, J. Li and H. Fang, Nature, 2017, 550, 380-383.

28 C. Leighton, Nat. Mater., 2019, 18, 13-18.

29 S. M. H. Michael J. Stephenson, and Robert A. W. Dryfe, Angew. Chem. Int. Edit., 2005, 44, 3075-3078.

30 Y. Zhao, C. Gao and B. Van der Bruggen, Nanoscale, 2019, 11, 2264-2274.

31 M. D. Fayer, Acc. Chem. Res., 2012, 45, 3-14.

$32 \mathrm{H}$. Niu, Y. Zhang, Y. Liu, B. Luo, N. Xin and W. Shi, J. Mater. Chem. A, 2019, 7, 8503-8509.

33 Y. Liu, H. Niu, W. Gu, X. Cai, B. Mao, D. Li and W. Shi, Chem. Eng. J., 2018, 339, 117-124.

34 Y. Liu, X. Cai, B. Luo, M. Yan, J. Jiang and W. Shi, Carbon, 2016, 107, 426-432.

35 Y. Liu, X. Gao, Z. Hong and W. Shi, J. Mater. Chem. A, 2016, 4, 8983-8988. 
Journal Name

36 Y. Zhao, J. Zhu, J. Ding, B. Van der Bruggen, J. Shen and C. Gao, J. Membrane Sci., 2018, 548, 81-90.

37 Y. Zhang, Y. Wan, G. Pan, X. Wei, Y. Li, H. Shi and Y. Liu, J. Membrane Sci., 2019, 573, 11-20.
38 J. E, Y. Jin, Y. Deng, W. Zuo, X. Zhao, D. Han, Q. Peng and Z. Zhang, Adv. Mater. Interfaces, 2018, 5, 1701052. 